\title{
Aeroacoustic analysis of the tonal noise of a large-scale radial blower.
}

\author{
Aurélien Marsan ${ }^{1 *}$, Stéphane Moreau ${ }^{1}$
}

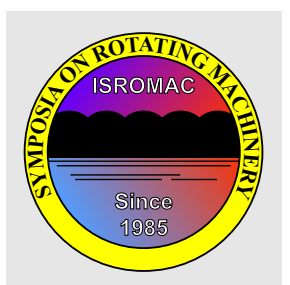

ISROMAC 2016

International

Symposium on

Transport

Phenomena and

Dynamics of

Rotating Machinery

Hawaii, Honolulu

April 10-15, 2016

\begin{abstract} and the trapdoors located on the volute sidewall. Some modifications of the geometry are suggested.

Keywords

Large-scale radial blower - Aero-acoustic - Unsteady RANS - acoustic analogy

${ }^{1}$ Department of Mechanical Engineering, University of Sherbrooke, Québec, Canada.
*Corresponding author: aurelien.marsan@usherbrooke.ca
\end{abstract}

Large-scale radial blowers are widely used in factories and are one of the main sources of noise. The present study aims at identifying the noise generation mechanisms in such a radial blower in order to suggest simple modifications that could be made in order to reduce the noise. The flow in a representative large-scale radial blower is investigated thanks to unsteady RANS numerical simulations. The radiated noise is calculated thanks to an inhouse propagation code based on the Ffowcs Williams-Hawkings analogy, SherFWH. The results highlight the main noise generation mechanisms, in particular the interaction between the rotating blades and the tongue, and the interaction between the rotating blades

\section{INTRODUCTION}

In most of manufacturing plants, the various air circuits are powered by large-scale radial blowers, the diameters of which can be as much as 2 or 3 meters. These blowers have to provide high volume flow rates, and they are sources of major acoustic disturbances for both workers in the factory and the neighbouring inhabitants, especially because of their very basic design. This noise can disturb the communications between the workers and make the production process more complex. It can also lead to health issues in the long run, besides seriously disrupting the living comfort around the factory.

The noise generated by centrifugal compressors is often dominated by the tones at the blade passage frequency and higher harmonics. This is a well known results, which is due to the strong interaction between the flow at the outlet of the rotor and the stator blades or the volute tongue. A spatial distorsion of the inlet flow can also contribute to the tonal noise. [1]

Previous studies about centrifugal blowers have shown that some modifications of the shape of the volute tongue could lead to a significant decrease of the tonal noise level. For a centrifugal blower, Embleton [2] has shown that sloping the edge of the tongue relative to the blade trailing edge reduces the level of the tonal noise by $12 \mathrm{~dB}$. On another centrifugal blower, Lyons et al. [3] have shown a similar effect. Thanks to the skewing of the volute tongue across several blade-toblade spacings, the authors report a decrease of the tonal noise by $10 \mathrm{~dB}$, without any loss in blower efficiency. They explained this reduction by a phase cancellation mechanism. More recently, Datong et al. [4] and Gu et al. [5] have experimentally confirmed that the modification of the shape of the tongue is an efficicient way for decreasing the tonal noise radiated by centrifugal fans. On the same type of machine, Cai et al. [6] have proven that such a decrease of the tonal noise is adequatly predicted thanks to a URANS calculation with the Ansys CFX software and the use of an acoustic analogy theory in order to calculate the sound radiation.

Large-scale radial blowers exhibit high tonal noise at the blade passing frequency, the level of which increases with the rotationnal speed. When the production rate is limited by the air flow in the ventilation system, the rotational speed should be increased at its maximum. This can lead to a very high level of tonal noise. Control techniques such as the volute tongue skewing are then necessary, whithout any loss in efficiency in order to maintain the flow rate as high as possible.

The present paper deals with a very particular radial blower, actually used in a steel factory. The tonal-noise issue is addressed thanks to URANS simulations, together with an acoustic propagation code based on the Ffowcs Williams-Hawkings analogy. First, the test case and the available experimental data are presented. Then, the numerical strategy is exposed, and an analysis of the global flow patterns is performed. Finally, the acoustic radiation is analysed.

\section{TEST CASE}

The test case is a large-scale radial blower actually used in a steel factory in Québec, Canada. Its size is approximately 
3.2 meters. The outlet rotor wheel diameter is approximately $1.8 \mathrm{~m}$. The width of the volute is 0.9 meter. The blade-tongue clearance is $0.19 \mathrm{~m}$. This blower is located upstream of a vertical chimney which guides the air outside, through the roof of the building. A schematic sketch of the aeraulic circuit is given in figure 1 .

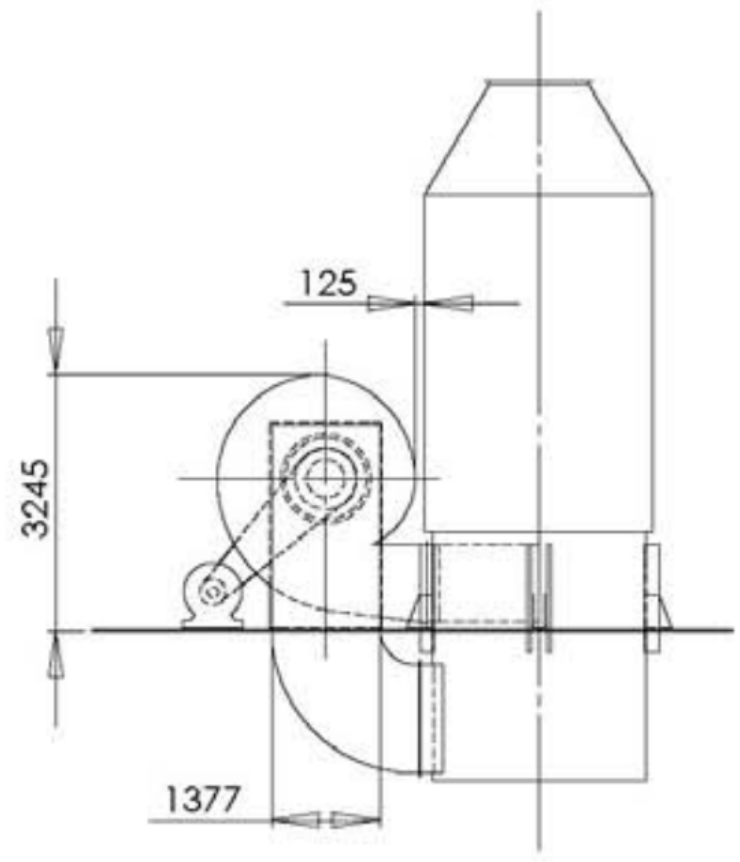

Figure 1. Global view of the aeraulic circuit. Dimensions are given in $\mathrm{mm}$.

A more precise 3D view of the blower geometry is shown in figure 2. It is composed of a rectangular inlet, a rotor and a volute. The rotor blades are purely radial. For structural reasons, they are maintained together thanks to two transverse plates. Trapdoors are located on the endwall of the volute. They introduce an azimuthal heterogeneity and can then contribute to the noise generation.

The rotational speed varies up to $1000 \mathrm{rpm}$, depending on the air flow which is necessary for the production rate. Brief experimental data obtained during maintenance operations indicate that the standard flow rate is $47500 \pm 5 \% \mathrm{~m}^{3} / \mathrm{h}$ at $900 \mathrm{RPM}$, and between $52000 \pm 5 \% \mathrm{~m}^{3} / \mathrm{h}$ at $1000 \mathrm{rpm}$. For this two rotationnal speeds, figure 3 shows the noise level measured at the outlet of the chimney, on the roof of the buidling. A peak is clearly visible at the blade passing frequency, which corresponds to an annoying siren noise. It is higher at 1000 RPM and is the point of this study. Unsteady RANS calculations should allow to identify the origin of that tonal noise, in order to suggest some control strategy.

\section{NUMERICAL SIMULATIONS}

Steady and unsteady RANS simulations have been performed in order to predict the flow within the blower. Steady-state

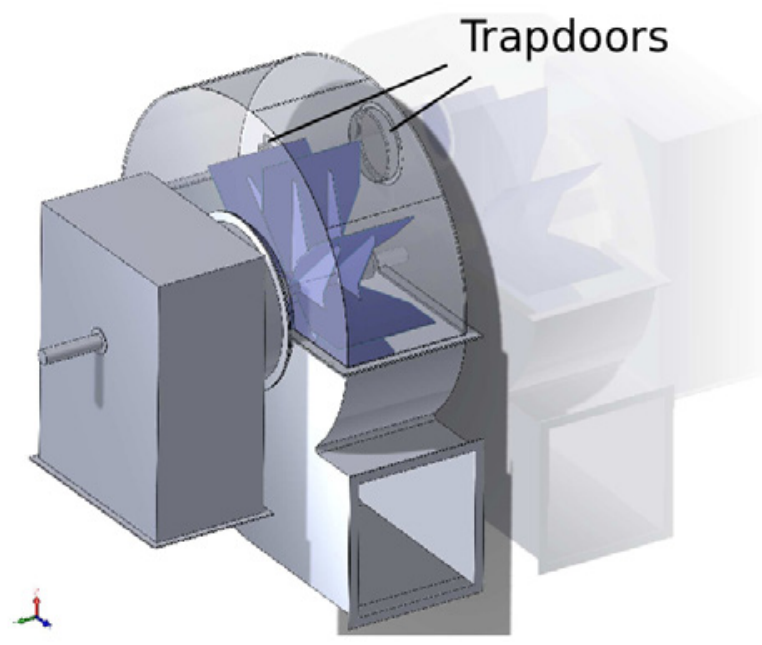

(a) Global view

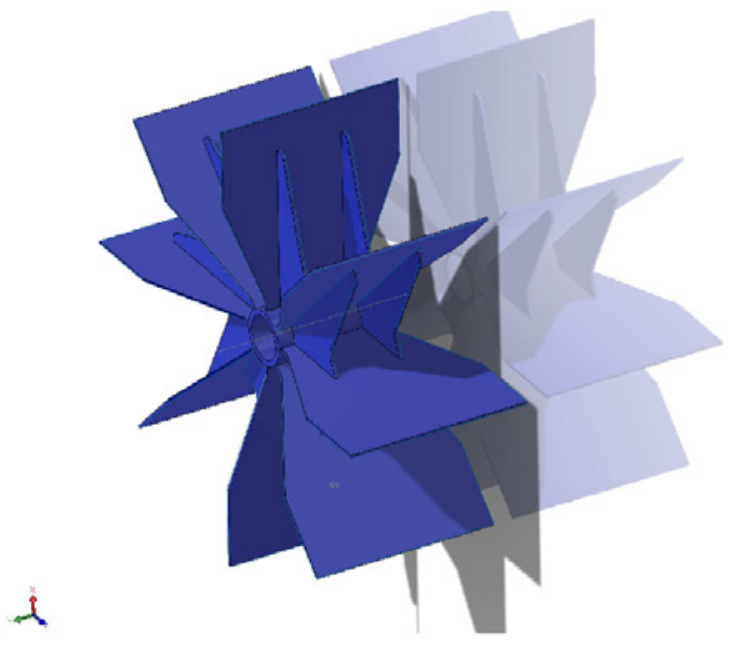

(b) Impeller

Figure 2. View of the test case

numerical simulations are more affordable. They allow to describe the whole operating range of the blower for the 900 RPM and 1000 RPM and to study the evolution of the main flow structures depending on the operating points. Unsteady numerical simulations are far more time consuming, and give access to the temporal evolution of the flow. In particular, the instantaneous wall pressure fields allows computing the acoustic radiation of the blower using an acoustic propagation code. Two operating points at 900 RPM and 1000 RPM are calculated with this model.

Various phenomena are possibly responsible for the experimentally observed tonal noise, as for example the inlet flow distorsion, the rotor-tongue interaction, and the interaction between the rotating blades and the trapdoors. The numerical results should provide some insight into the dominant source of the siren noise.

All simulations have been performed using the cluster Mammouth serie 2. 


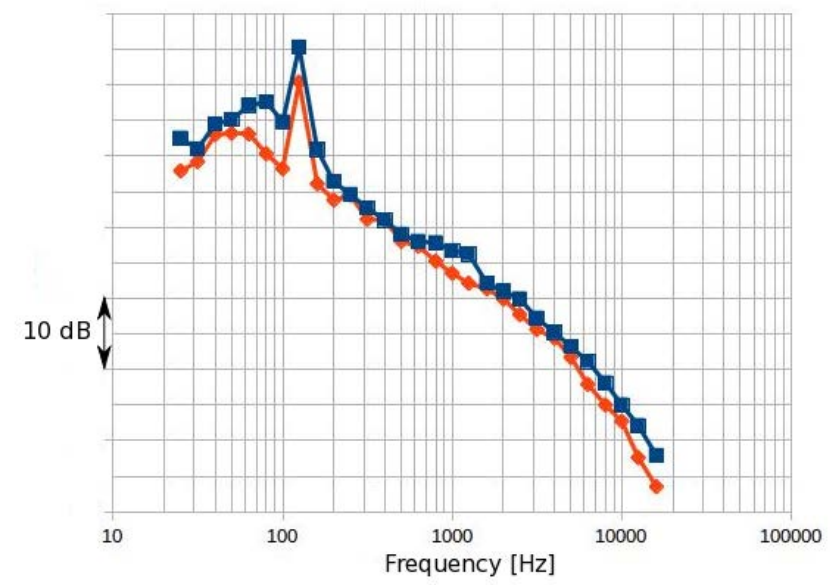

Figure 3. Sound pressure level at the chimney outlet $[\mathrm{dB}]-$ 900 RPM (red) - 1000 RPM (blue)

\subsection{Numerical model}

Numerical simulations have all been performed using the commercial CFD code ANSYS CFX R15.0. This code solves the three-dimensionnal Navier-Stokes equations on an unstructured grid, using an implicit element-based finite-volume formulation, and a pressure-based coupled algorithm solver. The flow is modeled as fully turbulent incompressible air. The effect of the turbulence on the mean flow is modeled using the shear-stress transport (SST) $\mathrm{k}-\omega$ turbulence model [7].

The incompressible model give access to the unsteady wall pressure fluctuations that are due to the aerodynamics, but does not take into account any propagation effet of the acoustic waves. It then discards the influence of reflexions and distorsions on the wall pressure fluctuations. This assumption is suitable for large wavelengths compared to the size of the surfaces, i.e. compact surfaces. Here, with a reference length of 1 meter, the source can be considered as semi-compact for frequencies less than $2 \mathrm{kHz}$. The blade passing frequency beeing equal to $133 \mathrm{~Hz}$, the assumption of compactness is suitable for the first harmonics of the BPF.

The unstructured mesh has been built using the software Centaur 10.05. The immediate vicinity of the solid walls is meshed with prisms whereas the volume is meshed using tetrahedrons. This ensures a good discretization of the boundary layers, the first cell width being set to $1 \mathrm{~mm}$. This value ensures a dimensionless wall distance $y^{+}$below 10 over all solid surfaces, except in the vicinity of the volute tongue where it reaches its maximum being equal to 10 .

Figure 4 shows the 3 domains used for the meshing. The inlet box domain counts 841000 cells. The rotor domains includes only one blade passage and counts approximately 1.7 millions of cells. The volute domain counts 7.2 millions of cells.

The mixing-plane approach is used for the steady-state numerical simulations. It is based on a circumferential averaging of the flow at the rotor-stator interfaces. This allows reducing the computational domain to one blade passage only in the rotor. This numerical approach is used in order to obtain a

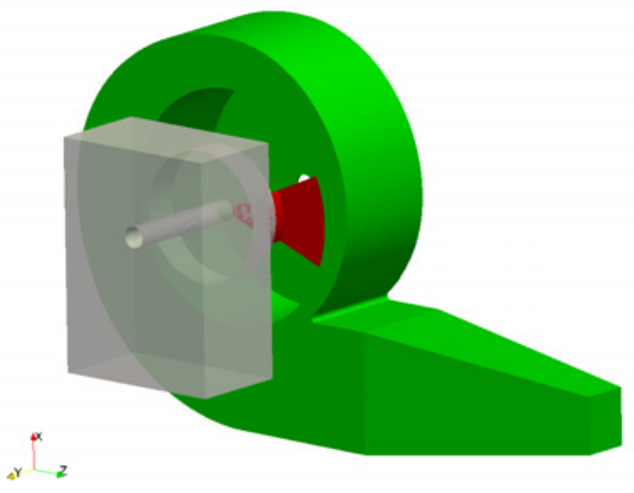

Figure 4. View of the mesh domains.

first mean flow field, which is afterwards given as initial flow field for the unsteady numerical simulation.

As concerns the unsteady numerical simulations, the mesh of the rotor domain is duplicated in order to reconstitute the full wheel and the rotor-stator interfaces are now modeled using the sliding mesh approach. The total mesh counts then approximately 17 millions of cells. Advance in time is performed using first-order backward Euler temporal discretization, and with 40 time steps per blade passage.

All solid surfaces are modeled as smooth walls using a no-slip boundary condition. Uniform total pressure and temperature are applied at the inlet. No detailled experimental data are available and a 5 percent turbulence rate has been set at the inlet. Regarding the outlet, a constant static pressure is applied.

\subsection{Steady-state numerical results}

Figure 5 shows the performance curves predicted by the steady-state numerical model for the $900 \mathrm{rpm}$ and $1000 \mathrm{rpm}$ rotational speeds. The static pressure rise through the blower stage is plotted as a function of the volumic flow rate. As expected, the flow rate increases with the rotational speed, and the static pressure rise decreases with the flow rate. The black dotted line stands for the square of the flow rate and passes through the $1000 \mathrm{rpm}$ operating point at $52000 \mathrm{~m}^{3} / \mathrm{h}$. It corresponds to an estimation of the pressure losses through the air circuit. It crosses the $900 \mathrm{rpm}$ operating line at a volume flow equal to $47000 \mathrm{~m}^{3} / \mathrm{h}$ approximately, which is in accordance with the experimental volume flow measured at this rotational speed.

In addition to the previous observations, a change in the slope of the operating line is visible for both rotational speed. This corresponds to a change in the flow regime or in the flow pattern. Figure 6 shows the curves of the inlet-to-outlet pressure rises through the inlet box, the rotor and the volute. The change of slope is obvious for the volute, whereas the slopes of the rotor and inlet curves are almost zero. The shape of the performance curve can then be entirely attributed to the volute, which is then the component responsible for the change of the pressure rise with the flow rate. The pressure 


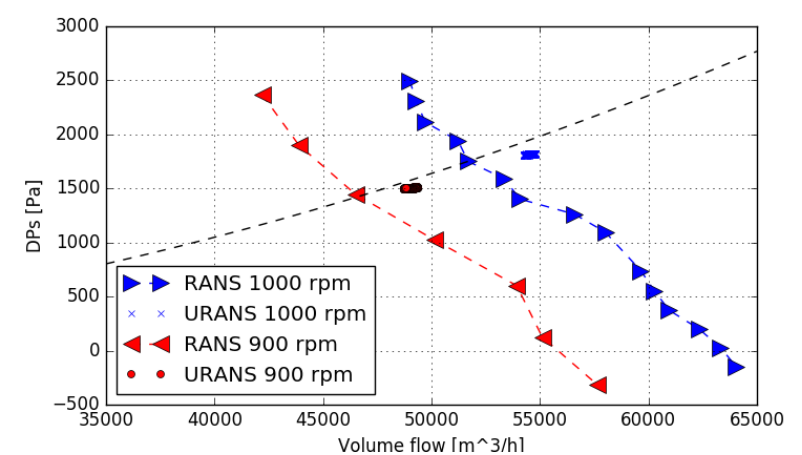

Figure 5. Performance curves - 900 RPM and 1000 RPM

rise in the rotor only depends on the rotational speed. And the pressure rise through the inlet block is constant.

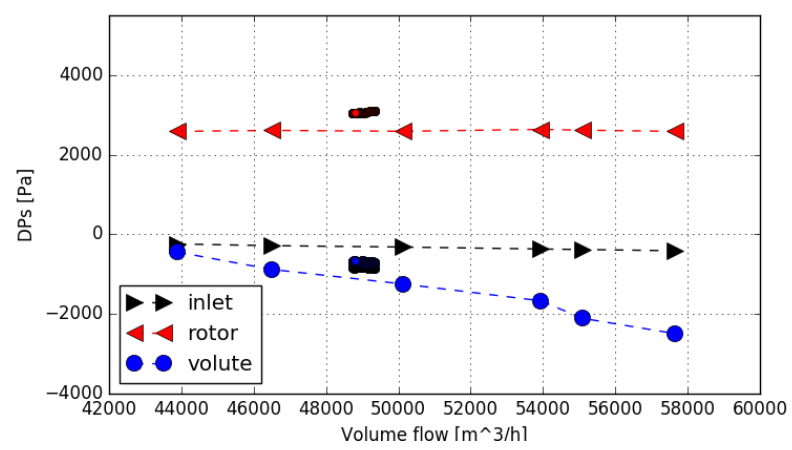

(a) $900 \mathrm{RPM}$

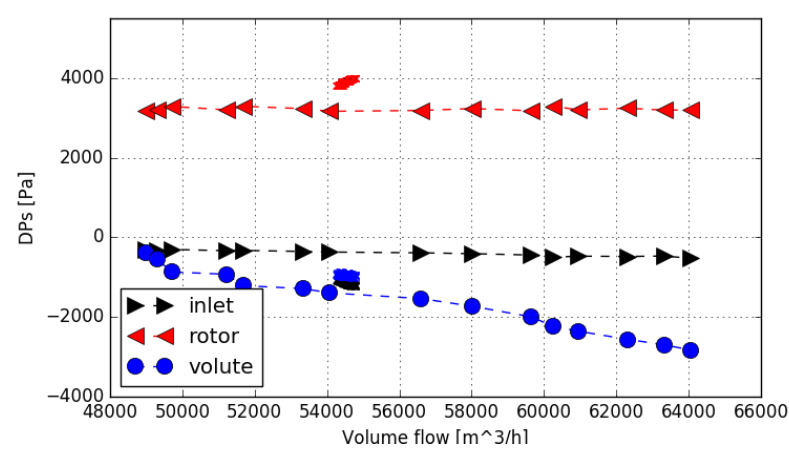

(b) 1000 RPM

Figure 6. Pressure rise through the components of the blower: inlet block, rotor and volute.

In order to give understanding of the change in the structure of the flow which explains the change of slope, figure 7 shows the flow pattern at mid-plane of the volute. Streamlines of the projected absolute velocity vector field are shown using the Surface Line Integral Convolution technique [8, 9, 10]. They are coloured by the magnitude of the absolute velocity field. The Surface Line Integral Convolution technique relies on the blurring of a noise function in the direction of the vector fields's integral curves, and is available in the open-source visualization software Paraview [11].

For high volume flow rate, figure $7 \mathrm{a}$, the radial component of the velocity in the centrifugal part of the blower as well as the axial component of the velocity in the outlet pipe of the volute are important. The stagnation point is located in the fluid volume, and most of the flow goes through the lower half of the outlet duct. The radial component of the velocity at impeller outlet decreases together with the flow rate, as well as the axial velocity in the outlet pipe. The flow becomes more and more tangential, and impacts the wall in the outlet duct of the volute. Below $60000 \mathrm{~m}^{3} / \mathrm{h}$, figure $7 \mathrm{~b}$, the stagnation point is located at the wall just downstream of the volute tongue. Then, the more the flow rate decreases, the more the flow becomes tangential and the more the stagnation point moves upstream. At $49000 \mathrm{~m}^{3} / \mathrm{h}$, figure $7 \mathrm{c}$, the stagnation point has moved upstream of the tongue and a massive backflow exists, which compromises the stability of the numerical model.

Then, three types of flow pattern can exist in the volute and may lead to different noise levels. In this case, the study focuses on the left part of the characteristic where the experimental operating point is located.

\subsection{Unsteady numerical results}

A full wheel unsteady RANS simulation has been performed in order to model the two operating points at 900 and $1000 \mathrm{rpm}$ labeled in figure 5. According to the present mesh and numerical model, each unsteady operating point costs $2.10^{7} \mathrm{CPU}$ seconds approximately, against $5.10^{6}$ with the steady-state one. But they allow to study the wall pressure fluctuations, and give informations about the flow patterns the disturbing tonal noise at the blade passing frequency comes from. According to Ffowcs Williams and Hawkings' (FWH) analogy, dipole terms distributed over the surface of the body are indeed proportional to the fluctuations of the wall pressure.

The performance coefficients calculated from the unsteady numerical results are shown in figure 5. Each symbol corresponds to an instantanous flow field.

For both rotational speeds, the volume flow rate predicted by the unsteady numerical model is higher than the volume flow rate predicted by the steady-state model. Since boundary conditions have been kept exactly the same as for the steady-state calculations, this difference can only be due to the effect of the unsteadiness and to the differences between the mixing-plane approach and the full wheel sliding mesh approach. Despite this difference, the numerically predicted flow rate remain within the experimental uncertainty range of $\pm 5 \%$. The operating points remains also within the left part of the characteristic curve. In addition, the flow pattern in the volute is the same as predicted by the steady-state results, the stagnation point being located just downstream of the volute tongue, see figure $7 \mathrm{~b}$. These results can then be used in order to identify the main tonal noise generation mechanisms.

\subsection{Main flow structures}

Due to the rotation of the rotor, azimutal singularities in the flow or the geometry are likely to induce synchronous wall 


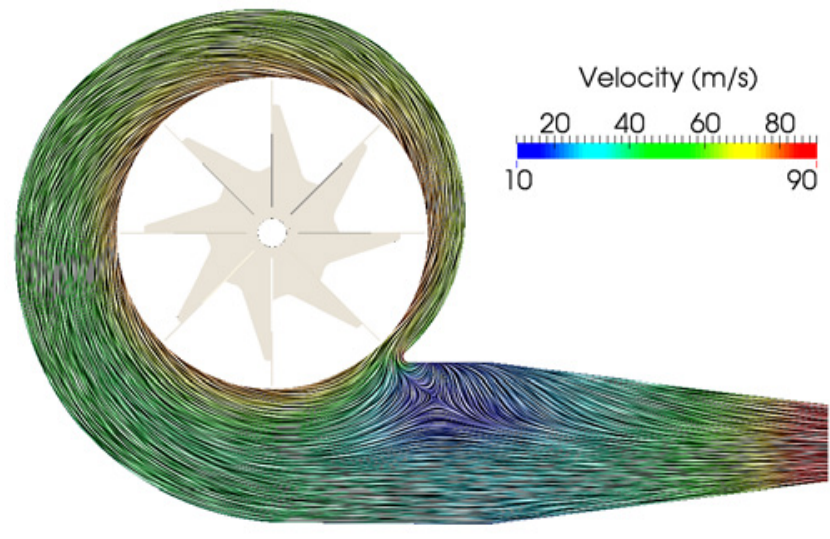

(a) $64000 \mathrm{~m}^{3} / \mathrm{h}$

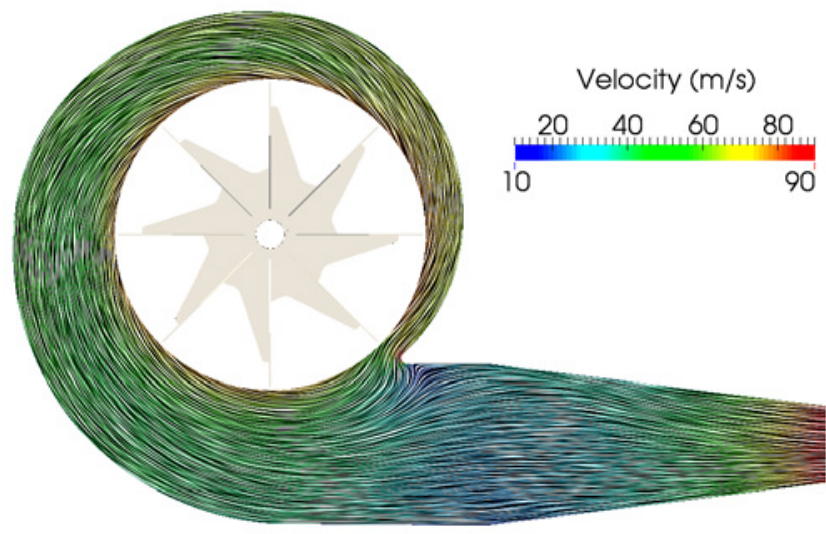

(b) $56000 \mathrm{~m}^{3} / \mathrm{h}$

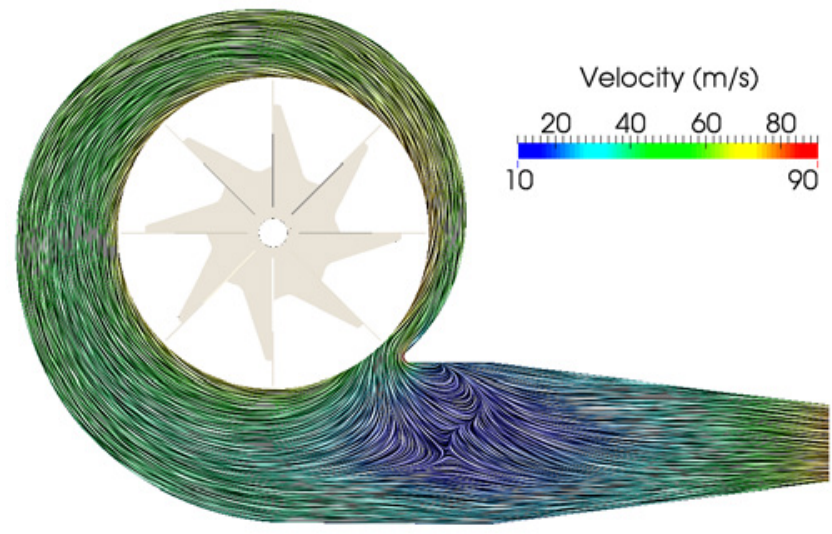

(c) $49000 \mathrm{~m}^{3} / \mathrm{h}$

Figure 7. Flow pattern at mid-plane of the volute - 1000 RPM RANS.

pressure fluctuation, which can contribute to the generation of the tonal noise at the first blade passing frequency. The analysis of the flow pattern has led to the identification of the four following main structures:

Inlet flow distorsion, figure 8 shows the absolute velocity magnitude at the inlet of the rotor. It highlights the flow distorsion that is mainly due to the shape of the air inlet box.
Blade-to-blade cavities For mechanical reasons, the rotor blades are held together by transverse plates. These plates forms cavities between the blades, and obstruct the air flow at the inlet. Figure 9a shows the magnitude of the relative velocity in a meridian plane in the rotor. It highlights the zone of very low velocity within the cavity. Figure $9 \mathrm{~b}$ shows the pressure in the median plane of the volute. The pressure within the cavities fluctuates over the rotor revolution under the influence of the volute tongue. The phenomenon generates wall pressure fluctuations that are synchronous with the rotor revolution and are likely to contribute to the tonal noise at the blade passage frequency.

Trapdoors cavities The trapdoors located on the backface of the volute also corresponds to cavities that interact with the rotating rotor blades. Figure 10a shows the pressure at rotor outlet, and figure $10 \mathrm{~b}$ shows the pressure field on the backwall of the volute. Singularities in the pressure field due to the trapdoors are clearly visible. They implie azimuthal distorsions in the pressure field due to a blockage effect. This is highlighted in figure 10a where high pressure areas are labeled.

Rotor-tongue interaction This phenomenon is a very wellknown effect in centrifugal compressors or blowers. The rotor blades interact with the volute tongue, which corresponds to a stagnation point. The presence of the tongue generates a local singularity in the azimutal pressure field, see figure $9 \mathrm{~b}$. The high pressure area in the vicinity of the volute tongue is also visible in figure 10a which shows the pressure field at rotor outlet. In this large scale radial blower, the radial gap between the rotor trailing edge and the volute tongue is important, approximately $15 \mathrm{~cm}$. The analysis of the wall pressure fluctuations will allow to determine if the rotor-tongue interaction is a major phenomenon in this kind of blower.

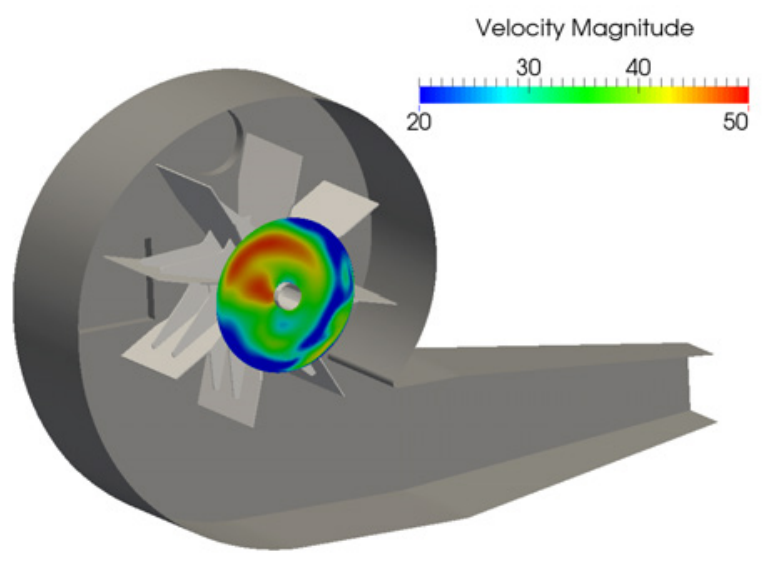

Figure 8. Inlet flow distorsion - 1000 RPM URANS. 


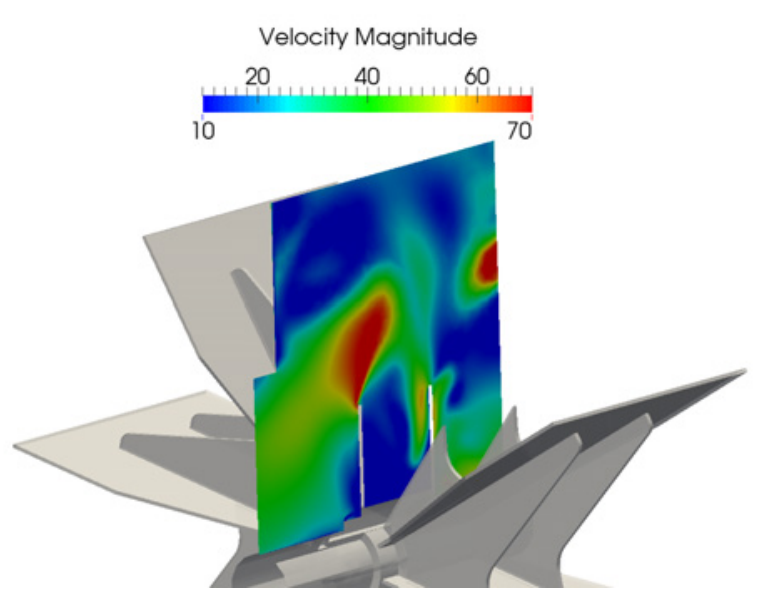

(a) Relative velocity magnitude in a meridian plane.

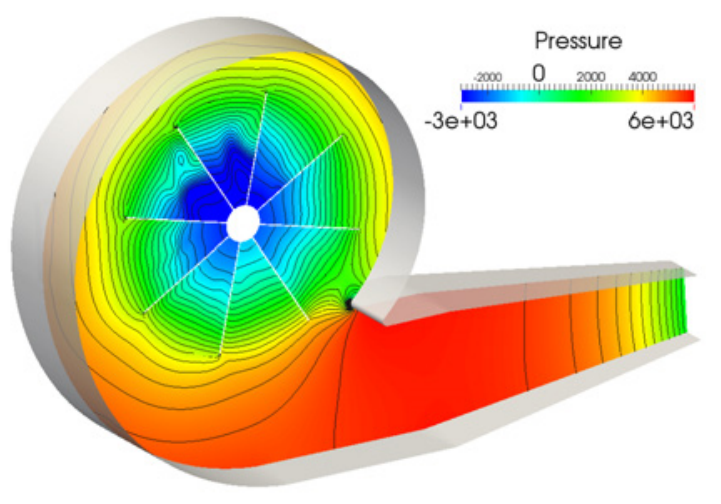

(b) Pressure in the mid-plane of the volute.

Figure 9. Influence of the rotor blade-to-blade cavities - 1000 RPM URANS

All these mechanisms can contribute to the generation of the tonal noise at the blade passing frequency.

\subsection{Wall pressure fluctuations}

In order to determine if one of the mechanisms is predominant, the analysis now focuses on the wall pressure fluctuations. The wall surfaces are divided in several parts, see figure 11. The contribution of each part is analyzed separately.

Figure 12 shows the maximum amplitude of the wall pressure fluctuations, for each surface and both rotational speeds. Peaks at the blade passing frequency and its harmonics are visible, except for the blade tip and root surfaces, the spectra of which are more broadband and do not show any emerging peaks. This is probably due to the very rough design of the rotor which leads to massive flow separations. The spectra are very similar at both rotational speeds, and there is no difference that could explain the $5 \mathrm{~dB}$ difference observed experimentally at the outlet of the chimney between the noise level at 900 RPM and 1000 RPM.

In decreasing order, the wall pressure fluctuations are maximum on the blade tip surfaces, the volute backwall sur-

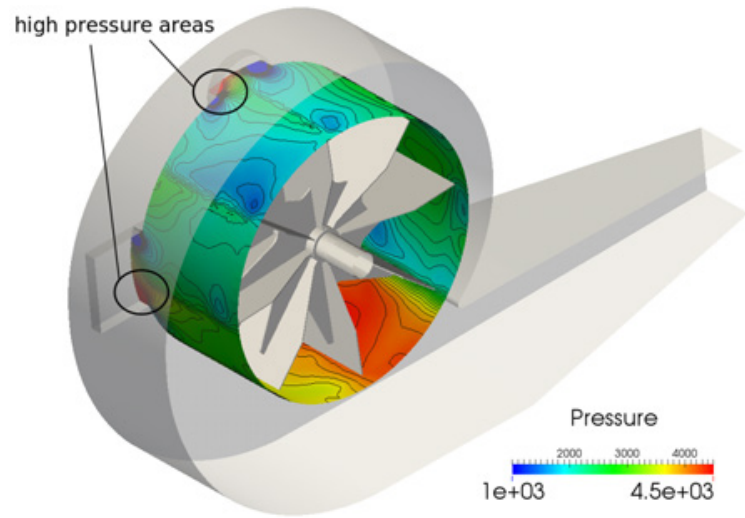

(a) Pressure at rotor outlet

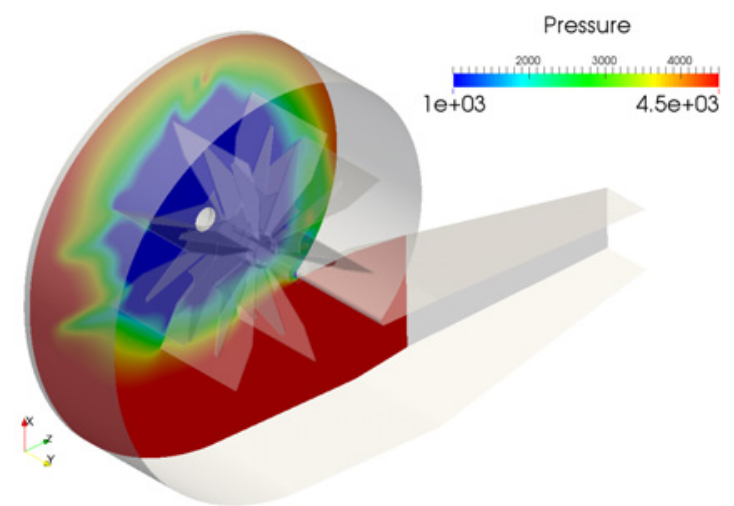

(b) Pressure on the backwall of the volute.

Figure 10. Influence of the trapdoors

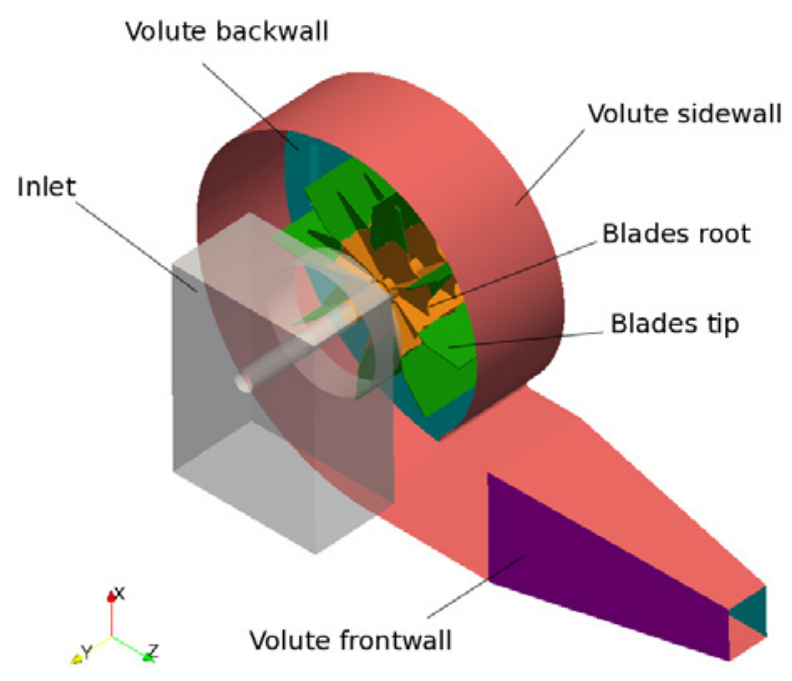

Figure 11. Division of the wall surface.

face, the blades root surfaces, the volute frontwall surface, the volute sidewall surface, and finally the inlet block surface. The wall pressure fluctuations are then higher on the volute 
backwall surface than on the volute tongue.

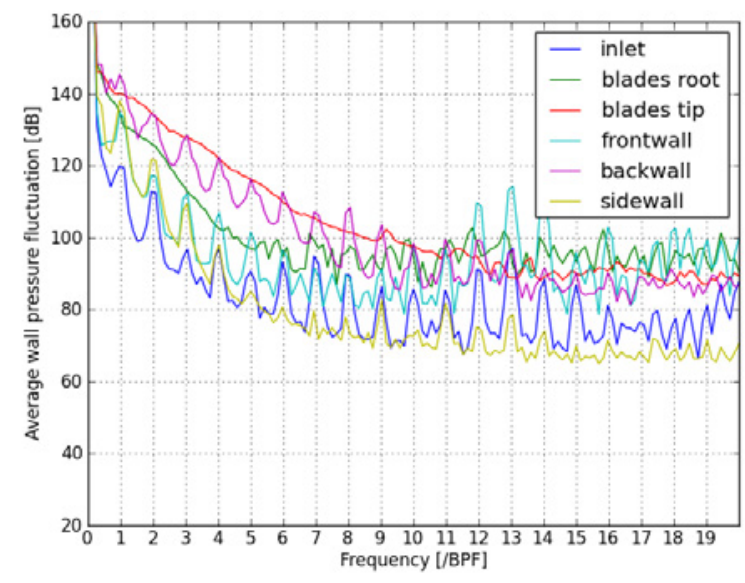

(a) $900 \mathrm{RPM}$

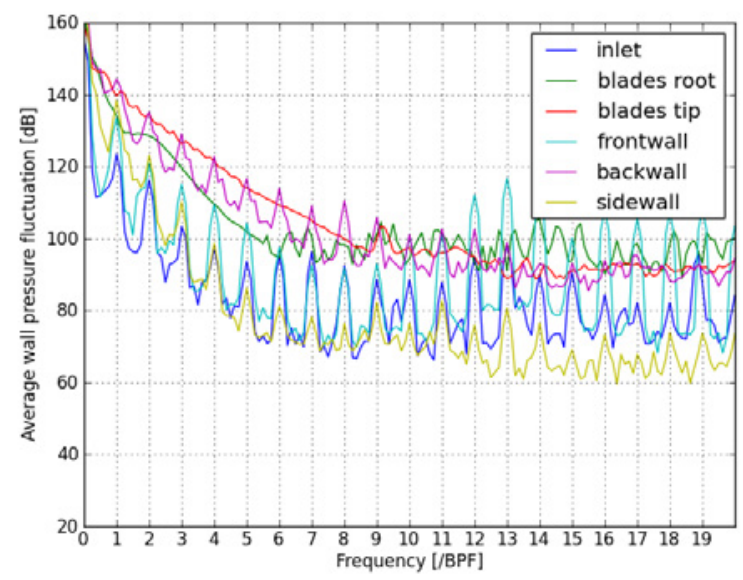

(b) 1000 RPM

Figure 12. Maximum amplitudes of wall pressure fluctuations.

Figure 13 aims at identifying the locations where the wall pressure flucuations are maximum. It shows the amplitude of the wall pressure fluctuations at $1 \mathrm{BPF}$, expressed in $\mathrm{dB}$. In the inlet block, the maximum amplitude is about $125 \mathrm{~dB}$ and is located in the vicinity of the rotor inlet. In the rotor, the maximum amplitude is higher, approximately $130 \mathrm{~dB}$. Three distinct patterns can be identified, which correspond to three separate phenomena:

Pressure wavefronts on the pressure side of the blades, which come from the edge of the first upstream transverse structural plate. The origin of this pressure pattern still remains unclear.

The trailing edge of the rotor blades. The location of this maximum can be explained by the interaction between the rotor and the volute tongue.

The back edge of the rotor blades. This location highlights the importance of the interaction between the blades and the trapdoors cavities.

In the volute, the maximum amplitude of the wall pressure fluctuations at $1 \mathrm{BPF}$ is $140 \mathrm{~dB}$. The maxima are located at the tongue and on the backwall, downstream of the trapdoors in the direction of the rotation.

\section{ACOUSTIC ANALYSIS}

In order to gain more insight into the acoustics, the sound radiated by the blower has been computed thanks to the inhouse acoustic propagation software, SherFWH. This code is based on the Ffowcs-Williams and Hawkings analogy. It has been used in order to compute the sound pressure level from the instantaneous pressure fields, on a sphere of 20 meters in diameter composed of 25 microphones. As previously, the wall pressure fields have been extracted during a full wheel revolution with a sampling frequency equal to 320 times the rotation frequency. This corresponds to 40 instants per blade passage. These parameters allow to compute the sound radiated by the blower up to $2600 \mathrm{~Hz}$ with a sampling equal to approximately $30 \mathrm{~Hz}$, the blade passing frequency beeing equal to $133 \mathrm{~Hz}$.

Figure 14 shows the sound power level calculated through the sphere, for the 900 RPM and the 1000 RPM operating points. As for the wall pressure fluctuations, both cases are very similar. This confirms that the numerical model is then not able to predict the $5 \mathrm{~dB}$ difference between the two rotationnal speeds. Further comparisons between the experimental and the numerical results should allow to understand this. For example, the propagation of the sound in the chimney may be included in the numerical model in order to gain understanding of its influence. The wall of the chimney is indeed made of an acoustic absorbant material, that could dump some frequencies more than others. However, the peak at the blade passing frequency is well predicted and very intense: $130 \mathrm{~dB}$ at $133 \mathrm{~Hz}$. Then, the sound power level decreases, and is below $100 \mathrm{~dB}$ for frequencies higher than $500 \mathrm{~Hz}$.

Figure 15 shows the contributions of the different elements to the sound power level. Only the 1000 RPM case is plotted since the 900 RPM case is again very similar.

The sidewall of the volute, including the volute tongue, is the main contributor to the peak at the BPF with a sound power level of $125 \mathrm{~dB}$. Then come the volute backwall and frontwall and the blade tip surface with a sound power level of $120 \mathrm{~dB}$, approximately. The amplitude of the peak at $1 \mathrm{BPF}$ for the inlet and the blade root are approximately $110 \mathrm{~dB}$ and their contribution to the tonal noise can then be neglected in comparison to the other elements.

Finally, figure 16 shows the directivity of the acoustic radiations for each surface elements. Since the outlet duct of the blower is aligned with the $\mathrm{z}$ axis, the sources which radiate more in this direction are subject to contribute more to the noise level at the outlet of the chimney. Since the main issue is the noise at the outlet of the chimney, this criterion should allow to precise which parts of the blower contribute 


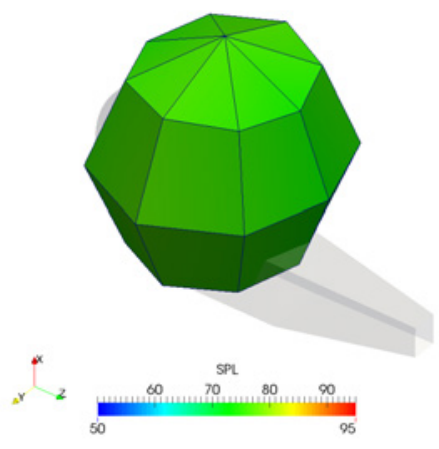

(a) Inlet

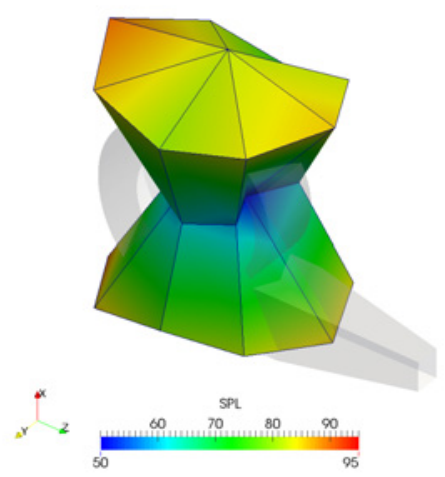

(d) Volute frontwall

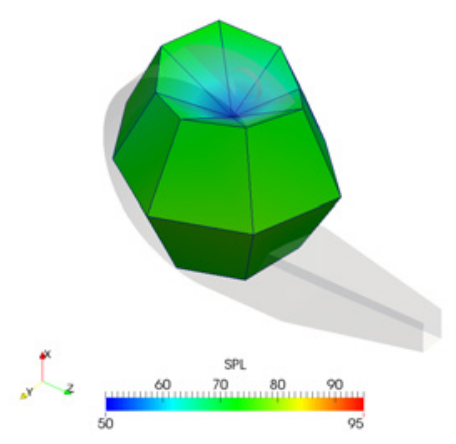

(b) Blades root

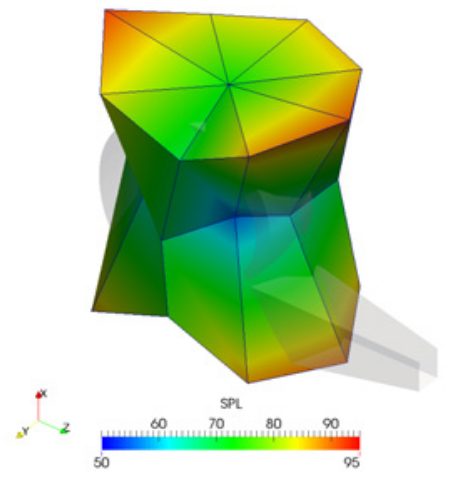

(e) Volute backwall

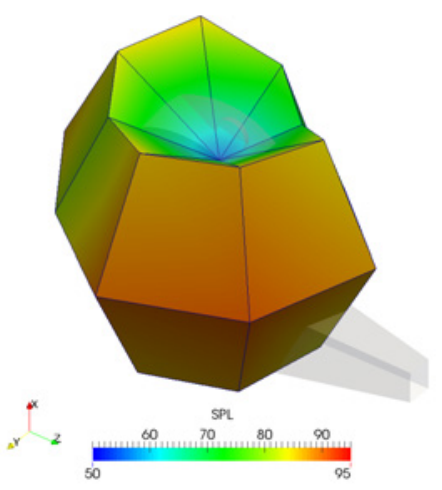

(c) Blades tip

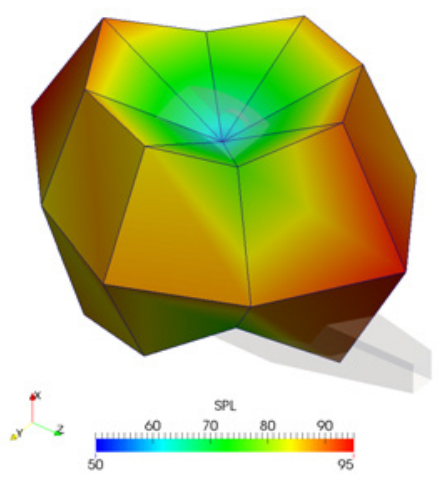

(f) Volute sidewall

Figure 16. Directivity of the acoustic radiation at the BPF.

the most. The directivity is shown as a deformable sphere, the radius of which varies as a function of the sound pressure level predicted at the microphone. The shadow of the blower, which has been enlarged in order to be visible, is shown in order to clarify the orientation of the view.

The radiation of the inlet block is isotropic, at a value of $75 \mathrm{~dB}$ approximately. It acts as as monopole, and is not the predominant source. As concerns the blade root, the sound pressure level is also relatively low. It remains below $80 \mathrm{~dB}$ and vanishes along the $\mathrm{X}$ axis. Again, the blade tip surface and the volute surfaces are found to be the predominant acoustic sources. The directivity patterns are very different. Volute front and back walls mainly radiates along the $\mathrm{X}$ axis, whereas the blade tip surfaces and the volute sidewall radiates in the YZ plane the same way as the blades root surface. The directivity associated with the volute frontwall and backwall are then unfavourable to the propagation of the sound in the outlet duct. The tip part of the blades and of the tongue of the volute are then the predominant sources as concerns the noise at the outlet of the chimney.

According to this study, the interaction between the volute tongue and the rotating blades is the main flow phenomenon that originate the high tonal noise at the outlet of the chimney. Acoustic treatments should then focus on this phenomenon in order to control the noise.

\section{CONCLUSIONS AND OUTLOOKS}

The present study gives a precious insight into the acoustics of a large scale radial blowers and suggests several acoustic treatments. Despite the very large spacing between the rotor trailing edge and the volute, the interaction between the rotating blades and the tongue has been identified as a major tonal noise generation mechanism, which is similar to the results obtained on centrifugal blowers. The front and back walls are also important acoustic sources, but the directivity of their radiation is unfavourable to the propagation in the outlet duct. They should however be taken into account in order to decrease the noise level within the building. In particular, the presence of the trapdoors on the backwall of the volute has been identified has a major source of tonal noise inside the building. This source can be easily controled by filling the cavities with some materials.

According to previous work in centrifugal blowers, the level of the tonal noise at the chimney outlet could be decreased by acting on the rotor-tongue interaction thanks to the skewing of the volute tongue. Further work will assess this hypothesis. In particular, the influence of this technique on the blower efficiency, and on the operating curves in general, will be numerically investigated. The volume flow rate should indeed be as high as possible, and an optimization procedure could be conducted.

Additionnal improvements could also include the design 

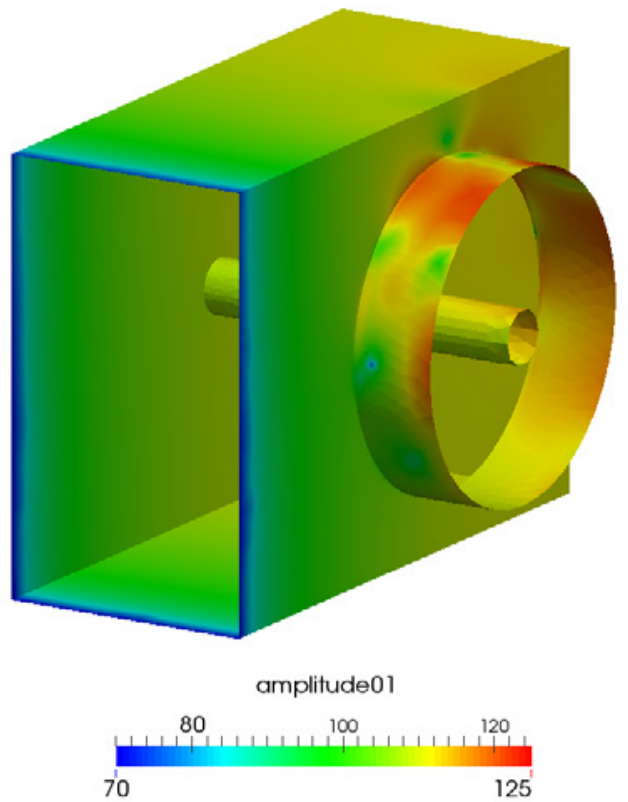

(a) Inlet block

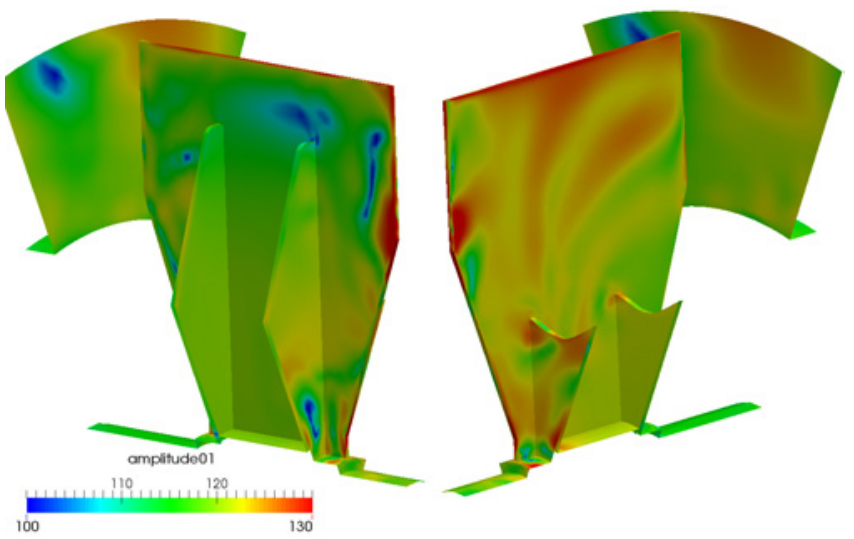

(b) Rotor blades

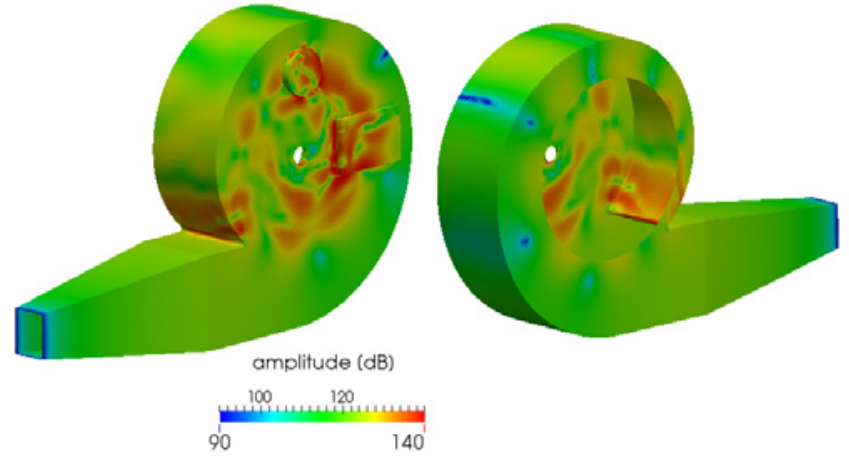

(c) Volute

Figure 13. Amplitudes of wall pressure fluctuations at 1BPF - 1000RPM

of an adequate inlet block in order to decrease the flow distorsion at the inlet of the rotor.

Finally, an analytical model well suited for this kind of geometry could be developped in order to allow a quick acoustic

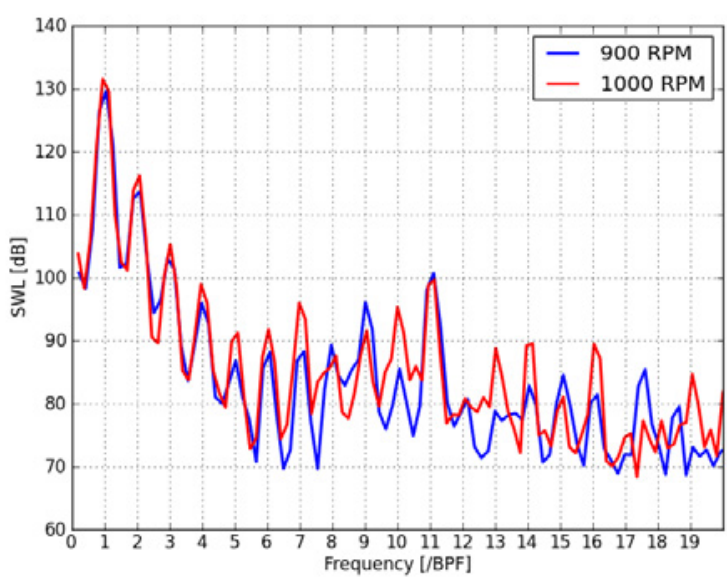

Figure 14. SWL

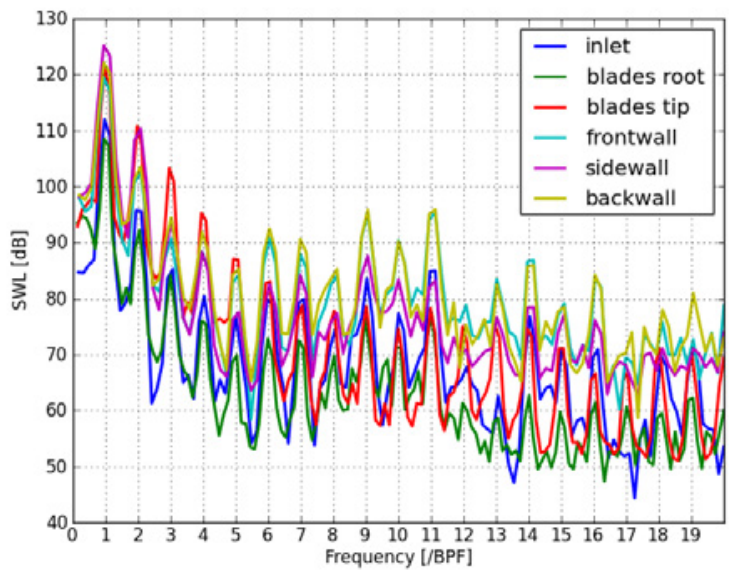

Figure 15. SWL - surface elements

diagnosis of any type of large-scale radial blower and suggest acoustic treatment.

\section{ACKNOWLEDGMENTS}

The authors also wish to thank the "Conseil de recherches en sciences naturelles et en génie du Canada" for promoting the collaboration between the Universities and the industries.

\section{REFERENCES}

[1] Jong-Soo Choi, Dennis K. McLaughlin, and Donald E. Thompson. Experiments on the unsteady flow field and noise generation in a centrifugal pump impeller. 263(3):493-514, 2003.

[2] T. F. W. Embleton. Experimental study of noise reduction in centrifugal blowers. 35(5):700-705, 1963.

[3] L. A. Lyons and S. Platter. Effect of cutoff configuration on pure tones generated by small centrifugal blowers. 35(9):1455-1456, 1963. 
[4] Qi Datong, Mao Yijun, Liu Xiaoliang, and Yuan Minjian. Experimental study on the noise reduction of an industrial forward-curved blades centrifugal fan. 70(8):1041-1050, 2009.

[5] Y. Gu, D. Qi, Y. Mao, and X. Wang. Theoretical and experimental studies on the noise control of centrifugal fans combining absorbing liner and inclined tongue. 225(6):789-801, 2011.

[6] Jiancheng Cai, Datong Qi, Fuan Lu, and Xuanfeng Wen. Study of tonal fan noise reduction by modification of the volute cutoff. 96(6):1115-1124, 2010.

[7] F. R. Menter. Two-equation eddy-viscosity turbulence models for engineering applications. 32(8), 1994.

[8] Brian Cabral and Leith Casey Leedom. Imaging vector fields using line integral convolution. In Proceedings of the 20th annual conference on Computer graphics and interactive techniques, pages 263-270. ACM, 1993.

[9] Henrik Battke, Detlev Stalling, and Hans-Christian Hege. Fast line integral convolution for arbitrary surfaces in 3d, visualization and mathematics: experiments, simulations and environments, 1997.

[10] Robert S Laramee, Bruno Jobard, and Helwig Hauser. Image space based visualization of unsteady flow on surfaces. In Proceedings of the 14th IEEE Visualization 2003 (VIS'03), page 18. IEEE Computer Society, 2003.

[11] Utkarsh Ayachit. The paraview guide: A parallel visualization application. 2015. 